\title{
L'école autrichienne contemporaine : un bref panorama
}

Die zeitgenössische österreichische Schule: ein kurzer Überblick

The contemporary Austrian school: a brief overview

\section{Christel Vivel}

\section{(2) OpenEdition}

Journals

Édition électronique

URL : https://journals.openedition.org/austriaca/1420

DOI : 10.4000 /austriaca. 1420

ISSN : 2729-0603

Éditeur

Presses universitaires de Rouen et du Havre

Édition imprimée

Date de publication : 1 juin 2020

Pagination : 197-214

ISBN : 979-10-240-1492-0

ISSN : 0396-4590

\section{Référence électronique}

Christel Vivel, «L'école autrichienne contemporaine : un bref panorama », Austriaca [En ligne], 90 |

2020, mis en ligne le 01 juin 2021, consulté le 05 octobre 2022. URL : http://journals.openedition.org/ austriaca/1420 ; DOI : https://doi.org/10.4000/austriaca.1420

Ce document a été généré automatiquement le 5 octobre 2022.

Tous droits réservés 


\title{
L'école autrichienne contemporaine : un bref panorama
}

\author{
Die zeitgenössische österreichische Schule: ein kurzer Überblick \\ The contemporary Austrian school: a brief overview
}

\author{
Christel Vivel
}

\section{Rappels historiques préliminaires}

1 L'histoire de l'école autrichienne d'économie fondée par Carl Menger (1840-1921) est jalonnée par différentes générations d'auteurs et de membres autoproclamés ou reconnus par leurs confrères au long des étapes de son développement. Carl Menger, l'un des trois "pères" de la "révolution marginaliste » est à l'origine de cette tradition, notamment en raison du débat sur les méthodes (Methodenstreit), qui l'opposa à l'école historique allemande, ainsi que, plus confidentiellement, ses travaux sur la monnaie des années 1890. Avec Menger, Friedrich von Wieser et Eugen von BöhmBawerk, ses cadets d'une dizaine d'années et ses disciples, ils constituent la première génération de ce qui devint avec eux une école de pensée au caractère spécifique.

2 L'école autrichienne est ensuite connue pour le renouveau qu'elle connut lors de la période de l'entre-deux-guerres, grâce aux travaux de ses représentants restés jusqu'à ce jour les plus célèbres, Ludwig von Mises, Friedrich von Hayek ainsi qu'un certain nombre d'autres auteurs dont la renommée connut des destins divers, parmi lesquels, entre autres, Oskar Morgenstern ou Fritz Machlup. La révolution keynésienne marqua la fin de l'heure de gloire des idées autrichiennes dans les années 1930. Si celle-ci semble perdre en notoriété en apparence, il n'en est cependant rien quant au fond des thèses débattues parmi les économistes professionnels. À la faveur de l'émigration de Mises et Hayek, une nouvelle génération de l'école autrichienne allait émerger. C'est l'histoire de ce renouveau que les pages suivantes présentent brièvement.

3 Si Menger, Wieser et Böhm-Bawerk sont les représentants majeurs de la première génération de l'école autrichienne, et Hayek et Mises ceux de la seconde, les auteurs 
auxquels nous nous intéressons correspondent donc à une troisième génération. Cette dernière n'a plus d'autrichienne que le nom puisque sa nationalité est essentiellement américaine. Elle est née suite à l'émigration de Mises et Hayek aux États-Unis. Son évolution est alors étroitement liée à l'organisation des universités et de la recherche aux États-Unis ${ }^{1}$.

\section{«Traversée du désert » et « grande traversée de l'océan » : l'émigration}

4 Après avoir enseigné à la London School of Economics, sur l'invitation de son directeur, Lionel Robbins, dans les années 1930, Hayek intègre en 1950 l'université de Chicago en tant que membre du Committee on Social Thought. N'appartenant ni au département d'économie (qui avait, semble-t-il, repoussé sa candidature), ni à ceux de gestion ou de droit, son influence était relativement limitée à cette époque sur le cursus académique local. Ce réel manque d'influence s'explique en outre par le fait que ses travaux tenaient alors essentiellement de la philosophie politique, et moins de l'économie que cela n'avait été le cas dans ses travaux de jeunesse sur les « cycles d'affaires » (business cycles) ou la théorie monétaire. Sa renommée et son influence sont cependant alors ainsi plus largement liées à ses publications plus récentes en philosophie politique, notamment son ouvrage $L a$ route de la servitude, pour lequel il réalise une tournée entre 1945 et 1950. La forme de renouveau que la tradition autrichienne connaît à cette occasion n'est donc liée ni à l'activité d'enseignement d'Hayek ni à des thèses identifiées comme proprement de leur domaine par les économistes de profession.

5 L'intégration de Ludwig von Mises dans la nouvelle terre d'élection américaine fut, quant à elle, encore plus difficile. À presque 60 ans, alors qu'il avait trouvé en Suisse une atmosphère point si différente de la Vienne de ses origines, il quitte en 1940 une position et une institution prestigieuses pour un nouveau pays sur lequel il n'a que peu de connaissances, même théoriques. Il ne devait retrouver un poste de professeur invité qu'après cinq ans de déboires, dans la toute jeune université de New York (New York University, NYU) qu'en 1945. Il a décrit ses premières années à New York comme très dures, financièrement et professionnellement tout à la fois. Ce sont ses articles pour le New York Times qui lui permirent de prendre contact avec le milieu du patronat américain, par le truchement de la National Association of Manufacturers ainsi que de la New York Chamber of Commerce.

Les propos de Mises concernant les dangers de l'interventionnisme et la nécessité de préserver et de promouvoir la liberté de l'économie de marché, la propriété privée et l'entrepreneuriat lui permirent de convaincre Léonard Read, directeur de cette Chambre de commerce, qui lui ouvrit les portes de la Foundation for Economic Education (FEE, Fondation pour l'Éducation économique) que Read avait créée dès 1946 dans la banlieue new-yorkaise, à Irvington-on-Hudson, loin des institutions centrales. Cette fondation financée par des fonds privés a encore aujourd'hui pour objectif d'enseigner aux jeunes générations les valeurs de la liberté individuelle, du marché libre, de l'entrepreneuriat et du respect absolu de la propriété privée. Les idées " autrichiennes" de Mises trouvèrent naturellement là un lieu d'écoute et, lui, de travail. En effet, pour développer des idées qui allaient prendre une coloration politique très conservatrice dans les États-Unis de la guerre froide, la fondation 
organise des séminaires; elle publie et diffuse des œuvres parmi lesquelles figurent bientôt celles de Mises et de Hayek.

7 Parallèlement, Mises a obtenu la charge d'un séminaire au sein du département de «business administration» de l'université de New York; ce séminaire devait être à l'origine du renouveau de l'économie autrichienne. Ce séminaire, dédié à l'écriture de mémoires de master ou de thèses de doctorat, regroupe en conséquence des étudiants, mais aussi de nombreux visiteurs, attirés par le discours anti-interventionniste de Mises, sa défense vigoureuse d'une économie de marché libre au moment où elle peut sembler contestée par de nombreux courants de pensée. Parmi les étudiants qui écoutèrent les leçons de Mises, citons par exemple (par ordre alphabétique par commodité) Bettina Bien-Greaves, Percy L. Greaves Jr., Henry Hazlitt, Israël Kirzner, Murray N. Rothbard, Hans F. Sennholz, Mary Homan-Sennholz ou Louis Spadaro.

8 Ces étudiants, parfois économistes, forment une jeune génération d'intellectuels qui allait contribuer à diffuser les idées autrichiennes, soit au travers de leur enseignement académique, soit grâce à l'action d'éducation et de promotion de ces mêmes idées par des fondations et des groupes de réflexion. Le renouveau de l'économie sur une base de concepts d'origine autrichienne allait se structurer au cours des années 1970, principalement autour de deux étudiants de Mises : Murray Rothbard, Israël Kirzner et d'un élève de Hayek au parcours atypique, Ludwig Lachmann. La section suivante les présente tous trois brièvement.

\section{La tradition autrichienne s'implante aux États-Unis : du long silence au revival}

9 Historiquement le renouveau de l'économie autrichienne intervint en raison de la conjonction de plusieurs événements : tout d'abord, il y eut la publication de divers travaux, dont ceux de Rothbard dès les années 1960 ; surtout, l'attribution du «prix Nobel d'économie ${ }^{2}$ » à Hayek en 1974 fut un coup de tonnerre; enfin, une succession de conférences fut organisée dans les années 1970 qui tranchait sur les activités militantes par un écho plus large et plus profond.

10 La première de ces conférences eut lieu en 1974 à South Royalton (dans le Vermont), où la cinquantaine de participants avait obtenu le soutien de l'Institut pour les études humaines ${ }^{3}$ pour écouter Kirzner, Rothbard et Lachmann. L'objet de la conférence était de caractériser la spécificité de l'économie autrichienne et les voies potentielles de son développement futur. L'année suivante, ce fut à Hartford (dans le Connecticut) que les jeunes chercheurs intéressés par l'économie autrichienne se réunirent puis, enfin, en 1976 au château de Windsor (en Écosse) ${ }^{4}$.

11 Ces conférences permirent aux participants de prendre conscience - si tel n'était pas déjà le cas - non seulement de leur différence par rapport aux thèses principales développées au sein de l'économie dite " orthodoxe » à l'époque (notamment le courant du «monétarisme » représenté en particulier par Milton Friedman), mais aussi de la nécessité de poursuivre un programme de recherches qui puisse se présenter comme en rupture totale avec les orientations d'un milieu académique jugé empreint de toutes les nuances $d u$ "progressisme" contre lequel la vaste majorité des membres s'inscrivait. Les contacts noués alors permirent de souder ceux qui devaient désormais se dire les héritiers véritables de la tradition autrichienne. Leur historienne, Karen 
Vaughn, parle de l'ouverture d'une véritable «croisade des économistes autrichiens pour montrer à Milton Friedman que ce sont les Autrichiens qui ont raison ${ }^{5}$ ».

Derrière cette apparente homogénéité se dissimulent toutefois de profonds désaccords entre ces nouveaux tenants de l'économie autrichienne revisitée, et ils vont eux-mêmes progressivement se scinder en plusieurs courants qui ne doivent avoir de cesse de s'opposer.

13 Cette scission prend essentiellement trois directions qui sont les voies de l'économie «néo-autrichienne» ou "autrichienne moderne» et dont chacune est de fait respectivement personnifiée par l'un des trois auteurs majeurs que sont Rothbard, Kirzner et Lachmann.

\section{Murray Rothbard (1926-1995)}

14 Diplômé en mathématiques, en économie et docteur en philosophie, Murray Rothbard fréquente le séminaire de Mises à l'université de New York alors qu'il est encore étudiant à l'université de Columbia. C'est par le biais de la Fondation pour l'éducation économique, déjà évoquée, que Rothbard a rencontré Mises, qui devient son mentor.

Les premiers travaux de Rothbard prennent prétexte de questions propres à l'histoire économique des États-Unis pour appliquer les préceptes misesiens. Dans America's Great Depression (1963), Rothbard conjugue un intérêt pour la théorie des cycles d'affaires telle que Mises la présente et la crise de 1929, qu'il explique comme la conséquence inévitable d'une politique d'expansion monétaire dans les années 1920 et de l'interventionnisme du gouvernement américain au moment où les premiers signes de crise se sont fait sentir. Rothbard se revendique de l'influence de la tradition autrichienne, mais c'est surtout de l'ouvrage de 1949, L'action humaine de Mises, qui prend à ses yeux en quelque sorte valeur de texte "sacré ", qu'il cherche à vulgariser les idées. Man, Economy and State, que Rothbard publie en 1962, se veut un traité d'économie fondé sur l'axiome fondamental de l'action humaine tel que Mises l'avait exposé.

16 L'ouvrage de Rothbard, qui se présente comme un traité, explique comment une économie de marché libre parvient à permettre à des individus libres de leurs choix de se coordonner mieux que ne les y autoriserait toute économie administrée fondée sur un système coercitif et interventionniste, ces deux notions allant nécessairement de pair. Tout en introduisant par endroits des représentations graphiques et des arguments empruntés à la microéconomie orthodoxe, Rothbard refuse de rentrer dans le moule du formalisme mathématique et il se fonde sur la méthode de la praxéologie avancée par Mises comme une logique générale de l'action humaine. Hormis les concordances basiques minimales avec la microéconomie, Rothbard avance des idées hétérodoxes à tous égards ou presque. Il en résulta non seulement un manque d'attention de la part des économistes de profession, mais aussi le sentiment plus diffus, qui allait se répandre parmi ceux-ci, que l'économie dite « autrichienne » était datée dans les conceptions qu'elle pouvait présenter et dans l'incapacité de représenter le monde moderne dans sa réalité et dans les standards formalisés atteints par la science économique contemporaine.

17 Le rôle de Rothbard dans le renouveau de la pensée autrichienne tient ainsi davantage à sa désaffection vis-à-vis du formalisme mathématique, à sa critique de la version dite "néoclassique» de la théorie de l'utilité ainsi que des conceptions, centrales pour 
l'orthodoxie, relatives à l'« équilibre économique ». La défense ardente de l'économie libre et de l'esprit d'entreprise par Rothbard lui vaut moins l'intérêt des économistes que des philosophes politiques qui se baptisent «libertariens». Inversement, l'interdisciplinarité inhérente à sa démarche et la capacité de l'économie " autrichienne " à mêler dans ses préoccupations science, économie et philosophie politique lui permirent d'attirer à sa suite de jeunes chercheurs.

$18 \mathrm{Au}$ fil du temps, les travaux de Rothbard quittèrent le terrain de l'économie pour évoquer davantage les questions historiques et politiques. Dans Power and Market, que Rothbard publie en 1970, il est, par exemple, expliqué comment la politique d'imposition publique remet en cause le principe de non-agression, et plus encore celui de la propriété libre et individuelle. À partir de cette époque, Rothbard affirme qu'une économie libre doit être une économie de propriétaires fonciers entièrement libres de céder leur propriété par le don, l'héritage ou l'échange sans que l'État ni toute autre institution de nature coercitive n'ait le droit de s'immiscer dans leurs affaires. Il adopte ainsi une position qui peut être qualifiée d' " anarcho-capitaliste ${ }^{6}$, en considérant que l'État viole par sa nature même les droits individuels et qu'il devrait dans tous les cas de figure imaginables laisser fonctionner les mécanismes de l'économie de marché, et « laisser-faire ». Rothbard s'en prend alors aux représentants de tous les courants de pensée visant à un objectif autre que celui-ci dans le domaine sociopolitique, et notamment très violemment aux influences philosophiques herméneutiques ou pragmatiques adversaires de ces positions ${ }^{7}$.

19 Au-delà de ses positions politiques et philosophiques libertariennes, Rothbard conserve un attachement pour les principes fondamentaux de l'économie autrichienne comme une certaine interprétation de l'individualisme subjectiviste (qui ne s'oppose pas à l'«objectivisme» de la méthode a priori qu'il reprend de Mises), une conception dynamique du processus de production, le rôle fondamental de l'entrepreneur (chez lui comme chez Mises, à l'évidence capitaliste) dans le fonctionnement régulier d'une économie de marché ${ }^{8}$. Ces éléments sont effectivement à l'origine du renouveau de la tradition autrichienne aux États-Unis.

\section{Israël Kirzner (né en 1930)}

20 Kirzner participa lui aussi au séminaire de Mises à l'université de New York, en 1955, dans le cadre de son diplôme de gestion (MBA). Il allait ensuite faire sa thèse de doctorat sous la direction de Mises, dont il prit la suite des enseignements à l'université de New York, où il est aujourd'hui professeur émérite ainsi qu'à la Fondation pour l'éducation économique. Dans les années 1960, Kirzner entreprenait de construire une théorie de l'activité entrepreneuriale qu'il souhaitait fonder sur les travaux de Menger, de Mises et de Hayek, en réalisant une véritable synthèse, qui fut parfois qualifiée de " voie médiane ${ }^{9}$ » au sein du renouveau de l'économie autrichienne.

21 Son premier ouvrage majeur sur le sujet, Competition and Entrepreneurship (1973), avait, comme dans le cas de Rothbard, pour objectif initial de faire connaître les travaux de Mises, mais aussi de Hayek, auprès de ses collègues économistes de profession. Cet ouvrage est aujourd'hui considéré comme un jalon majeur dans la littérature sur l'entrepreneur. Il allait être suivi de nombreux autres ${ }^{10}$, qui ont permis à Kirzner de préciser sa pensée sur le lien entre entrepreneuriat, processus de marché, monopole et propriété. 

leur oppose la notion de processus de marché, développée notamment dans The Meaning of Market Process (1992). Kirzner s'écarte de l'« orthodoxie " néoclassique en ce qu'il s'intéresse non pas à la relation entre prix et quantités caractérisant un équilibre de marché, mais à la manière dont il est possible de parvenir à un tel équilibre par un processus dynamique. Dans la version kirznerienne de l'orientation autrichienne comme à l'origine chez Menger, plutôt qu'à l'équilibre, c'est au processus qui permet de l'atteindre que l'économiste doit s'intéresser.

Renouant également avec la définition hayekienne de l'équilibre, Kirzner réhabilite l'entrepreneur. Il reprend en cela les caractérisations données par Menger et Mises. L'entrepreneur devient la force motrice du processus de marché. Face à l'incertitude qui entoure le déroulement et l'issue du processus de production, l'entrepreneur découvre et exploite les opportunités de profit qu'il a su repérer. Il n'est donc pas simplement calculateur, comme le propose la théorie néoclassique, mais c'est un acteur qui juge et qui agit, qui anticipe et qui a les bonnes idées en faisant preuve de clairvoyance et de prévoyance (foresight).

'entrepreneur est attentif, c'est-à-dire vigilant aux évolutions du processus de marché : il exerce son œil averti pour capter des opportunités de profit. Capable d'apprendre, de modifier ses plans lorsque se produit un changement, il réagit et il devient lui-même une source du changement. L'entrepreneur contribue par son action à disséminer de l'information aux autres participants. L'activité entrepreneuriale diffuse aux autres participants des informations déjà existantes, mais ni alors «connues » ni exploitées. L'entrepreneur exerce une certaine persuasion sur les autres participants au marché. Il est celui qui introduit à la fois une nouvelle opportunité et qui, en même temps, fournit une connaissance de l'existence de cette opportunité. Il persuade ainsi d'autres acteurs de le suivre: il convainc ses fournisseurs de lui faire confiance, son personnel d'accepter de suivre sa stratégie, ses clients de se fier à la qualité des produits, et ainsi de suite.

Dès lors, l'activité entrepreneuriale a pour effet de réduire l'ignorance. Elle est " équilibrante ", puisqu'elle tend à améliorer la coordination des actions des différents participants au marché. L'entrepreneur-découvreur, l'entrepreneur kirznerien, est un acteur fondamental dans la poursuite du processus de marché.

\section{Ludwig Lachmann (1906-1990)}

Élève de Werner Sombart à l'université de Berlin et d'Emil Kauder, historien de l'utilité marginale qui redécouvre les archives de Menger au Japon ${ }^{11}$, Lachmann s'intéresse à la conception autrichienne des cycles et du capital. Il se pose ainsi naturellement en élève de Hayek, lors du passage de ce dernier à la London School of Economics en 1933. Lachmann prend une place très particulière au sein de la tradition autrichienne. Il s'écarte aussi géographiquement des autres artisans du renouveau autrichien, puisqu'il part occuper un poste de professeur à l'université de Witwatersrand, en Afrique du Sud. Les travaux de Lachmann s'articulent autour des thèmes principaux suivants: un subjectivisme radical, l'importance du temps et du changement, une analyse en termes de processus de marché et le rôle de l'entrepreneur. Le programme de recherche autrichien est fondé sur le subjectivisme: les préférences et les anticipations sont subjectives. L'individu agit donc en environnement incertain en fonction de la 
représentation qu'il se fait de celui-ci ; en son sein, il agit en fonction de circonstances sur lesquelles il pense ne pas pouvoir agir.

Le subjectivisme individuel et l'incertitude fondamentale qui le sous-tend sont donc liés et inhérents à la nature même de l'économie dans la tradition autrichienne. Toutefois, Lachmann va plus loin en développant un subjectivisme qu'il veut radical. Selon lui, toute action est fondée sur un plan, c'est-à-dire une "image mentale » ou une « interprétation» de la réalité. Les moyens, les fins et les obstacles étant donnés, constituant la limite de l'action des êtres humains, cette action et, en conséquence, les choix des hommes dépendent de leur interprétation du passé et de leur représentation du futur, autrement dit de leurs anticipations. La réalisation d'un plan individuel dépend donc à la fois des anticipations et des expériences vécues dans le passé par ces individus. Ils poursuivent leurs plans simultanément, ils partagent certaines fins et certains moyens. Chaque plan individuel constitue un " point d'orientation " pour les plans des autres individus. Les «frictions » ou du moins les "problèmes d'interaction ou de coopération » apparaissent dans la confrontation des plans des participants au marché, conduisant à une situation de déséquilibre. Il n'est donc pas possible de prévoir a priori l'issue du processus de marché. Cette issue n'est plus un équilibre, comme chez Kirzner, mais un perpétuel déséquilibre, une forme de chaos qui n'a pas de raison de cesser.

Plus encore, pour Lachmann, il n'y a pas un seul, mais plusieurs processus de marché. Il en distingue trois: les "processus internes au marché » (intra-market processes), les "processus entre les marchés» (inter-market processes) et les "processus macroéconomiques »(macroeconomic processes $)^{12}$.

Les processus « intra-marché » renvoient au fonctionnement des marchés particuliers où offreurs et demandeurs se coordonnent directement (par exemple, le marché aux légumes). Ce type de marché se complexifie du fait que des intermédiaires - les « marchands »- peuvent apparaître pour réguler le marché. Le marchand fixe alors luimême des prix pour les acheteurs (ou consommateurs) et les vendeurs (appelés ici des « commerçants »).

31 Les processus inter-marchés renvoient, quant à eux, à des marchés non équilibrés où un excès d'offre sur l'un d'eux peut permettre de combler un excès de demande sur un autre. On n'atteint une position d'équilibre que dans de très rares cas particuliers; l'existence de biens de substitution ou de stocks peut notamment alors expliquer l'absence de tout équilibre. Enfin, les processus macroéconomiques sont des processus «inter-marchés» demeurant en mouvement perpétuel. Les processus de multiplication, d'expansion ou de contraction de l'activité économique se composent d'une série de phénomènes de ce type. L'issue des processus de marché étant indéterminée, les individus font face à une situation d'incertitude radicale, selon le terme proposé par Lachmann.

32 Comment expliquer alors que les individus continuent d'agir et ne perdent pas pied face à cette incertitude? Dans une société complexe où les actions des divers individus dépendent de leurs anticipations et du résultat pour chacun de son interaction avec les autres, les individus sont cependant capables de prendre des décisions en se fondant sur leur interprétation de la situation et sur leur anticipation du futur. Ils s'appuient pour cela sur des «schémas d'orientation » qui ne sont rien d'autre que les institutions elles-mêmes. Lachmann établit alors un pont avec la tradition institutionnaliste qui 
allait à son tour être explorée par certains représentants de la tradition autrichienne dans les années $1990^{13}$.

\section{La tradition autrichienne contemporaine}

33 À partir des années 1980, les représentants de cette tradition autrichienne renouvelée se sont penchés sur l'héritage dont ils étaient les descendants lointains. Ils ont tenté de se positionner par rapport à l'orthodoxie néoclassique, d'une part, mais aussi par rapport à l'hétérodoxie montante de l'institutionnalisme qui vivait un renouveau aux États-Unis tout en étant issu des divers courants, allemand historiciste et américain "vieil institutionnaliste", de la fin du XIx siècle. Par ailleurs, des membres de la tradition autrichienne contemporaine, les héritiers de Rothbard en particulier, se sont employés à revenir sur certains débats fondateurs de la tradition autrichienne, comme le débat des années 1920 sur la possibilité de réaliser un calcul économique dans une économie socialiste.

34 À la faveur de ces débats renouvelés, des économistes "autrichiens » américains, comme Joseph T. Salerno ou Peter G. Klein pour ne citer que les plus actifs, ont affirmé que la tradition autrichienne contemporaine était divisée en deux tendances à bien distinguer. La première orientation est héritière des travaux de Wieser et de Hayek et elle se retrouve autour des travaux de Kirzner pour mettre en évidence le subjectivisme, le processus de marché, le rôle informationnel des prix et la tendance équilibrante de l'entrepreneur-découvreur d'opportunités. La seconde tendance, plutôt héritière de Böhm-Bawerk et de Mises, se retrouve autour des efforts de Rothbard pour considérer que la tradition autrichienne doit sa spécificité à ce que Klein décrit comme " "l'économie banale" de la théorie des prix, du capital, de la monnaie, des cycles et de l'interventionnisme ${ }^{14} »$. Dans cette représentation simplifiée, voire simpliste de la tradition autrichienne, l'influence de Lachmann est occultée.

La tradition autrichienne contemporaine s'est intéressée de manière grandissante à des questions d'économie politique, telles que celle de la banque libre (free banking) avec le problème des réserves dites "fractionnaires" (à savoir, dans quelle mesure les réquisits de réserves bancaires s'imposent-ils ?). Cette analyse fondée sur la philosophie dite "libertarienne" - ou «libertaire", terme qui a toutefois des connotations distinctes en philosophie politique - s'est développée notamment par les efforts du Mises Institute (fondé en Alabama) ou à la Fondation pour l'éducation économique, déjà mentionnée supra. Ces deux institutions ont fait la promotion d'une banque "libre", c'est-à-dire d'un système où la création monétaire n'est pas le monopole de l'État. La banque «libre", au fonctionnement analogue à toute entreprise commerciale, émettrait des supports monétaires privés en fonction du montant des réserves (fractionnaires) dont elle dispose, sans pouvoir déroger à cette règle de prorata.

Ces auteurs critiquent le caractère inflationniste du système monétaire international ; ils le remettent en question dans une tradition misesienne et hayekienne au nom des " principes traditionnels du droit civil et commercial, selon lesquels chaque individu ou chaque entreprise doit remplir ses obligations en strict accord avec la lettre de ce qui a été établi dans chaque contrat ${ }^{15}$. » Derrière cette conception de l'économie bancaire se profile une nouvelle critique à l'encontre de l'économie administrée et de tout interventionnisme gouvernemental. Dans la période qui s'ouvre alors, les auteurs de la tradition autrichienne se concentrent davantage sur ces questions d'économie 
politique, notamment en particulier celle de la légitimité de faire référence à l'équilibre. Les travaux de Gerald O'Driscoll (Université d'État de l'Iowa) et de Mario Rizzo (Université de New York) sur le temps et l'incertitude radicale sont à noter dans un paysage où le renouveau des travaux au sein de la tradition "autrichienneaméricaine " s'organise autour de plusieurs centres de recherche et instituts.

Le Mises Institute, mentionné plus haut et rattaché à l'université d'Auburn située en Alabama, est sans doute l'institut le mieux connu aujourd'hui des centres autrichiens et il figure parmi les principaux instituts dédiés à la pensée dite «libérale». Fondé par Rothbard et longtemps dirigé par L. H. Rockwell Jr., cet institut se veut un centre de recherches et d'enseignement du libéralisme classique et des idées autrichiennes, dans une pure tradition d'orthodoxie suivant les leçons de Mises et de Rothbard. Il est engagé dans la « défense de l'économie de marché, la propriété privée et des relations internationales pacifiques ${ }^{16}$.» Il édite nombre d'ouvrages et des revues dites "autrichiennes" selon son objectif statutaire de promouvoir l'enseignement et la recherche issus de la tradition de Mises et de sa continuation par Rothbard. Strictement orienté pour développer un des courants "néo-autrichiens ", il s'inscrit plus largement dans la lignée des fondations dédiées à la liberté individuelle et à la promotion du rejet de l'interventionnisme gouvernemental.

Les activités du Mises Institute se prolongent au travers d'un autre institut de droit privé, à la fois groupe de réflexion et laboratoire des idées dites «libertariennes ", l'Institut Caton (du nom du penseur romain). Basé à Washington, le Cato Institute est la version la " plus politiquement engagée » du Mises Institute, assumant un rôle de lobby. Parallèlement, d'autres centres de recherche dédiés à la tradition autrichienne, mais moins engagés sur des positions libertariennes, ont vu le jour, et l'Institut pour les études humaines (Institute for Humane Studies) est une autre institution de promotion des idées libertariennes, qui a succédé au Volker Fund comme sponsor de la tradition autrichienne ${ }^{17}$.

Une autre institution avec des branches à l'international, les Hayek Center s'intéressent à l'œuvre de Hayek, pour la diffuser et la développer en s'inscrivant dans une démarche d'histoire de la pensée économique. La Société du Mont Pèlerin » avait été fondée dès 1951 autour de Hayek et des ordolibéraux allemands dont Hayek était proche (notamment Wilhelm Röpke) dans la foulée de l'après-Seconde Guerre mondiale et de la montée de la guerre froide. Ses membres, bien que ne partageant pas toujours les mêmes points de vue, ont en commun qu'ils «perçoivent les dangers de l'expansion du gouvernement, non seulement sous la forme de l'État-providence, mais au travers du pouvoir des syndicats, des monopoles industriels et dans la menace de l'inflation ${ }^{18}$ ». Son objectif est, encore aujourd'hui, de promouvoir le libéralisme politique et économique et de lutter contre sa remise en cause.

Sur le plan académique, des programmes de recherche autrichiens fleurissent dans différentes universités américaines au sein des départements d'économie. Le plus remarquable est sans doute le programme d'économie autrichienne développé au sein du département d'économie du College des sciences humaines et sociales de l'université George Mason, où sont notamment intervenus Kirzner, Rizzo, James Buchanan ${ }^{19}$, Larry White, Roger Garrison ou Peter Boettke. Le Center for Market Process ${ }^{20}$ est quant à lui un centre de recherche qui se consacre à une recherche académique sur l'étude du fonctionnement d'un marché décentralisé. 
41 Au sein de l'université de New York, un programme d'économie autrichienne s'est développé, le programme sur les "fondements de l'économie de marché ", sous la responsabilité de Rizzo et David Harper ${ }^{21}$. Des séminaires et des conférences sont organisés tout au long de l'année universitaire. Le séminaire d'été accueille des étudiants américains et étrangers dont le séjour est bien souvent combiné avec les séminaires de la Fondation pour l'éducation économique à Irvington-on-Hudson.

L'intérêt croissant pour les idées autrichiennes a en outre conduit un certain nombre d'universitaires intéressés par le développement de la pensée autrichienne à fonder la "Société pour le développement de l'économie autrichienne» (Society for the Development of Austrian Economics, SDAE), une association de chercheurs et d'universitaires contribuant à répandre l'« économie autrichienne». Sa première présidente fut Karen Vaughn et l'association organise notamment une session dédiée à la tradition autrichienne dans le cadre de la conférence annuelle de la très connue Southern Economic Association.

Cet essor a eu pour conséquence de redéployer progressivement la tradition autrichienne au-delà du sol américain. Quatre facteurs majeurs ont joué à cet égard : d'abord le développement de ces différents instituts, puis la multiplication des publications sur la libre entreprise, l'activité entrepreneuriale et l'analyse du fonctionnement de l'économie de marché, ainsi que la dynamique créée par et dans les universités américaines autour de ces thèmes et, enfin, un contexte politique international favorable pendant environ deux décennies.

Ce redéploiement a à son tour impacté la tradition libérale européenne, qui était la source originelle de ces idées par les travaux des fondateurs de l'école, mais que ses représentants contemporains avaient largement oublié. Ce qui a grandi, s'est nourri et a participé à l'émergence de la tradition autrichienne contemporaine, c'est une version américanisée - où les textes (même les textes fondateurs) ne sont plus lus ni compris en allemand, mais dans leur version en anglais américain. En France, l'étude de la tradition autrichienne s'est implantée en particulier à l'université d'Aix-Marseille et à l'université Paris Dauphine, grâce à l'activité de chercheurs intéressés par la pensée libérale, à l'origine européenne.

Le Journal des économistes et des études humaines est publié depuis 2005 par De Gruyter pour perpétuer la tradition libérale française; il entend retrouver l'origine qu'était le courant né de sa création sous le titre de Journal des économistes en 1841. À partir des années 1980, un certain nombre d'économistes comme Florin Aftalion, Jacques Garello, Henri Lepage et Pascal Salin se sont intéressés à la théorie économique autrichienne, en adoptant un point de vue plus ou moins critique.

La tradition autrichienne a ainsi connu un renouvellement, non seulement par des travaux en histoire de la pensée économique, mais aussi grâce à des contributions théoriques en économie, dans la lignée de son développement aux États-Unis. Les idées libérales se diffusent en France au travers de l'enseignement universitaire, de la recherche, mais aussi de formes variées d'activisme. Des fondations et des associations participent de ce mouvement militant à des niveaux divers ${ }^{22}$.

Depuis le début des années 2000, en prenant inversement position sur un plan expressément strictement académique et en faveur d'une redécouverte de l'héritage proprement européen de la tradition autrichienne, Gilles Campagnolo propose de retrouver la pensée autrichienne des origines au moyen d'un programme de 
présentation et de traduction en français des œuvres phares de Menger, qui sont restées sans version dans notre langue durant près d'un siècle et demi. Campagnolo a notamment produit les traductions de ses Recherches sur la méthode (1883-2008) et des Principes d'économie politique (1871-2020). Il a également donné la seule biographie intellectuelle en français du fondateur de l'école autrichienne ainsi que de nombreux articles en français, en anglais, en allemand, visant notamment à expliquer les raisons de la désaffection française concernant les conceptions autrichiennes authentiques.

D'autres initiatives, en Europe et ailleurs, permettent à la tradition autrichienne de "survivre». Dans le monde hispanophone, Jesús Huerta de Soto, professeur à l'université Juan Carlos de Madrid, œuvre pour la tradition autrichienne en proposant un master dédié à l'économie autrichienne. Il a aussi contribué à la diffusion et au développement $d u$ programme de recherche de cette tradition dans sa version contemporaine en créant le Journal of Market Processes ainsi qu'en organisant des conférences annuelles. Le dilemme d'avoir à choisir entre une activité strictement académique et des formes variées de militantisme se pose à tous les acteurs de la pensée économique autrichienne contemporaine.

\section{Conclusion}

49 La tradition autrichienne contemporaine, héritière de Menger, Mises et Hayek, s'intéresse aujourd'hui encore à la théorie des cycles et au free banking, nous le disions.

Toutefois, le renouveau de l'école autrichienne est passé (et devra encore trouver) d'autres voies de développement que la réitération des enseignements des fondateurs de l'école. Le renouveau dont elle apparaît clairement en quête, du moins lorsqu'elle ne réoriente pas ses efforts vers le militantisme idéologique et politique, s'appuie sur le positionnement subjectiviste, les analyses du marché en termes de processus, le rôle informationnel des prix et la tendance « équilibrante » de l'action entrepreneuriale. Les économistes qui se revendiquent (encore) de la tradition autrichienne tentent de souligner l'apport des enseignements des auteurs fondateurs à des questions contemporaines comme la neuro-économie, la "Net-économie » ou les systèmes financiers parallèles (monnaies cryptées, banques parallèles, etc.)

51 Plus certainement, comme l'écrivait Boettke dès 1994, l'avenir de la tradition autrichienne passe par une analyse interdisciplinaire permettant de se placer sous l'angle de l'analyse autrichienne pour comprendre l'activité entrepreneuriale et ses rapports avec les autres fonctions économiques. L'activité entrepreneuriale est alors regardée comme un instrument analytique pour cerner à la fois les frontières et la nature des activités de la firme capitaliste, les stratégies de développement des entreprises. Des économistes comme Frédérique Sautet, élève et coauteur de Kirzner, mais aussi Harper, Nicolas Foss ou Todd H. Chiles travaillent en ce sens.

52 Les frontières du programme de recherche «autrichien» sont ainsi fouillées et repoussées, donnant lieu à de vifs débats sur la légitimité de son rapprochement avec la théorie néo-institutionnaliste ${ }^{23}$, les représentations de l'entrepreneur « autrichien ${ }^{24}$ ", voire avec les théories managériales en entrepreneuriat ${ }^{25}$ (Chiles 2010). Il faut encore attendre pour que ces travaux en cours chez des auteurs sympathisants de la tradition autrichienne fassent à leur tour l'objet d'une analyse concernant les développements de la tradition autrichienne. 


\section{BIBLIOGRAPHIE}

KIRZNER Israel M., Ludwig von Mises. The Man and His Economics, Wilmington, ISI Books, «Library of Modern Thinkers ", 2001.

KIRZNER Israel M., Competition and Entrepreneurship, Chicago, University of Chicago Press, 1973.

ROTHBARD Murray N., " Man, Economy and the State, a treatise on economic principles ", dans Man, Economy and the State with Power and Market, Auburn, Ludwig von Mises Institute, 2004, p. 1-1029.

\section{NOTES}

1. Cette histoire se déroule donc en terre d'exil pour les représentants de l'école « autrichienne » des origines, proche du Middle West (Chicago notamment) et loin de la Mitteleuropa. Cette école "néo-autrichienne », parfois dénommée "austro-américaine», ne constitue pas le thème du présent volume, mais il est impossible d'en taire ni la réalité ni l'importance historique; les références que lui font les autres textes de ce volume suffisent amplement à le montrer. Voir aussi l'introduction générale de ce numéro. [N.D.L.R.]

2. Il s'agit en fait du " prix de la Banque de Suède en sciences économiques en mémoire d'Alfred Nobel». Ce prix récompense des contributions exceptionnelles en sciences économiques. Il se distingue des autres prix Nobel en ce qu'il est géré par la Fondation Nobel, mais qu'il n'a pas été créé par le testament d'Alfred Nobel. Il faut ajouter que le comité qui choisit Hayek prit la précaution de désigner également Gunnar Myrdal, dont les options «suédoises " et "socialesdémocrates " se démarquaient point par point de celles de Hayek. L'indication donnée n'était donc pas idéologique, mais méthodologique : des considérations d'ordres méthodologique, social, voire philosophique pouvaient légitimement pénétrer la réflexion économique.

3. Voir plus loin infra, pour plus d'informations détaillées, sur l'Institute for Humane Studies.

4. Cette dernière conférence donna lieu à la publication en 1978 par Louis Spadaro d'un ouvrage collectif, New Directions in Austrian Economics, Kansas City, Sheed Andrews and McMeel, 1979.

5. «[That was] a crusade to show Milton Friedman that Austrian economics is good economics» (Karen I. Vaughn, Austrian Economics in America, The Migration of a Tradition, Cambridge, Cambridge University Press, 1998, p. 111).

6. De même, dans un sens opposé, mais ayant en commun la détestation de l'État, il avait existé un « anarcho-syndicalisme » à la fin du XIX ${ }^{\mathrm{e}}$ siècle, qui était contemporain de l'essor de l'école autrichienne des origines.

7. Nous renvoyons à cet égard le lecteur francophone à l'ouvrage de Gilles Campagnolo, paru en 2006, sur la critique de l'influence de la théorie conversationnelle et de la french philosophy, notamment, sur les campus des universités américaines. Dans "Seuls les extrémistes sont cohérents." Rothbard et l'École austro-américaine dans la querelle de l'herméneutique, Lyon, ENS, "Économie politique moderne ", Campagnolo a traduit notamment le texte de Rothbard: "The Hermeneutical Invasion of Philosophy in Economics ", Review of Austrian Economics, vol. 3, $\mathrm{n}^{\circ} 1$, 1989, p. 45-60.

8. Nous renvoyons le lecteur à la communication effectuée lors de la XXI ${ }^{\mathrm{e}}$ conférence de l'European Society of History of Economic Thought par Gilles Campagnolo et Christel Vivel (2017) : « Kirzner and Rothbard on an Austrian theory of entrepreneurship: the heirs of both Menger and Mises discuss action and the role of institutions ». 
9. Nous empruntons l'expression à l'article de Roger Garrison, « Austrian economics as a middle ground: comment on Loasby ", dans Israel M. Kirzner (éd.) Method, Process and Austrian Economics, Lexington, D. C. Heath \& Co., 1982, p. 131.

10. Parmi les principaux ouvrages d'Israel Kirzner, notons Perception, Opportunity and Profit (1979), Discovery and the Capitalist Process (1985), et The Meaning of Market Process (1992).

11. Sur ce sujet, voir les travaux de Gilles Campagnolo, "La bibliothèque viennoise de Carl Menger conservée au Japon: étude des sources d'une pensée économique », Austriaca, $\mathrm{n}^{\circ}$ 50, octobre 2000, p. 173-197; "Un exemple de réception de l'économie occidentale au Japon: le Fonds Menger de l'Université Hitotsubashi », dans Nadine Lucas et Cécile Sakai (dir.), Japon Pluriel 4, Paris, Philippe Picquier, 2001, p. 211-221 ; Critiques de l'économie politique classique. Marx, Menger et l'École historique [2004], Paris, Matériologiques, « E-conomiques », 2014.

12. Ludwig Lachmann, The Market as an Economic Process, New York, Basil Blackwell, 1986, p. 6 et suiv.

13. Notons ce point essentiel et surprenant si l'on se souvient que l'école autrichienne est née chez Menger dans le conflit avec l'orientation dite "éthique » et, au fond, institutionnelle des historicistes allemands.

14. Texte original : " "mundane economics" - price theory, capital theory, monetary theory, business cycle theory, and the theory of interventionism " (Peter G. Klein, "The Mundane economics of the Austrian School », Quarterly Journal of Austrian Economics, vol. 11, n 3-4, 2008, p. 165).

15. Jesús Huerta de Soto, «Banque centrale ou banque libre : le débat théorique sur les réserves fractionnaires ", Journal des économistes et des études humaines, vol. 5, nº 2-3, 2017, p. 387.

16. Voir les statuts de l'Institut tels que présentés sur leur site internet www.mises.org.

17. Voir à ce propos la présentation critique qu'en fait Joseph Salerno : « The Rebirth of Austrian Economics - In Light of Austrian Economics », The Quarterly Journal of Austrian Economics, vol. 5, $\mathrm{n}^{\mathrm{o}} 4$, p. 118.

18. Cette citation est extraite des statuts de la Société du Mont-Pèlerin. Voir www.montpelerin.org.

19. James M. Buchanan est un sympathisant de la tradition autrichienne, mais il n'en est pas un représentant. Il a développé la Virginia School of Economics autour de la remise en question des politiques de choix public. Il est, à certains égards, critique des positions autrichiennes, tout cela dans un contexte purement américain.

20. Ce centre de recherches est rattaché à l'université George Mason. Ce centre fut rebaptisé James M. Buchanan Center for Political Economy au début des années 2000, puis récemment Mercatus Center.

21. L'université qui avait accueilli Mises, «NYU », se distingue bien de l'université Columbia, et elle se décrit elle-même comme « université privée dans le service public » (A private university in the public service).

22. Citons par exemple le rôle de l'institut Coppet qui œuvre pour la diffusion des œuvres des libéraux français.

23. Virgile Chassagnon et Christel Vivel, «Frank H. Knight and R. H. Coase on the theory of the firm », History of Economic Ideas, vol. 21, $n^{\circ} 2,2014$, p. 63-84.

24. Gilles Campagnolo et Christel Vivel (dir.), «Les figures de l'entrepreneur / The Entrepreneur: Facets of Yesterday and Today ». Revue de philosophie économique / Review of Economic Philosophy, vol. 15, n $\mathrm{n}^{\circ}$, juin 2014.

25. Todd H. Chiles et al., «Dynamic Creation: Extending the Radical Austrian Approach to Entrepreneurship ", Organization Studies, vol. 31, nº 1, 2010, p. 7-46. 


\section{RÉSUMÉS}

L'école autrichienne est connue pour sa théorie des prix, du capital, de la monnaie et des cycles d'affaires, moins pour sa conception de l'entrepreneuriat; elle fut pourtant développée des origines jusqu'à la «troisième » génération. L'analyse de l'action entrepreneuriale esquissée par les premières générations fut poursuivie dans l'émigration par Hayek et Mises, donnant naissance aux générations ultérieures d' « Autrichiens » (ou « Néo-autrichiens »). Trois auteurs portèrent alors le flambeau sur le sol américain : Murray Rothbard, Israël Kirzner et Ludwig Lachmann. Après le débat sur le calcul économique socialiste mené à travers le prisme des enseignements initiaux, ils proposèrent d'analyser l'économie de marché comme processus en soulignant le rôle de l'incertitude, de la subjectivité et de l'entrepreneur. Cette contribution présente dans une optique contemporaine cette troisième génération dont les thèmes sont repris par les spécialistes en entrepreneuriat et en gestion-management. La filiation avec la tradition mengérienne, misesienne et hayekienne est encore présente.

Die Österreichische Schule kennt man hauptsächlich auf Grund ihrer Theorien über die Preise, das Kapitals, die Währung und die Konjunkturzyklen, weniger wegen ihres Konzeptes des Unternehmertums. Dieses wurde aber sehr wohl seit den Anfängen bis zur „dritten“ Generation entwickelt. Die von den ersten Generationen skizzierte Analyse von unternehmerischer Tätigkeit wurde in der Emigration von Hayek und Mises fortgesetzt, sodass eine weitere Generationen von „Österreichern“ (oder „Neo-Österreichern“) entstanden ist. Drei Autoren wurden zu ihren „Fackelträgern“ auf amerikanischem Boden: Murray Rothbard, Israēl Kirzner und Ludwig Lachmann. Nach der Diskussion über das ökonomische Kalkül des Sozialismus, die im Brennpunkt der anfänglichen Lehren geführt wurde, schlugen sie vor, die Ökonomie des Marktes als einen Prozess zu analysieren, wobei sie die Rolle der Unsicherheit, der Subjektivität und des Unternehmers hervorhoben. Der vorliegende Artikel präsentiert unter einem zeitgenössischen Aspekt diese dritte Generation, deren Themen von Spezialisten in den Fächern Betriebswirtschaft und Verwaltung-Management wieder aufgenommen werden. Die Verbindung zur Tradition von Menger, Mises und Hayek ist noch immer präsent.

The Austrian School of Economics is recognized for its price theory, capital theories and representation of money and business cycles. Its conception of entrepreneurship is less known. The analysis of entrepreneurship was initiated by the founding fathers but has been developed and more systematically theorized by the pupils of Mises and Hayek after their emigration and settlement in the United States of America. Three authors take over Austrian economics on the American soil: Murray Rothbard, Israël Kirzner and Ludwig Lachmann. Analyzing the economy of this time through the prism of the teachings of Austrian Economics, they came back on the calculation debate and propose a market process analysis of economics. Moreover, they underline the role of uncertainty, the entrepreneur and subjectivity. In this way they opened a new path for the study of entrepreneurship and management theories focusing on the discovery of profit opportunity and the process of entrepreneurship. The present paper aims to present the third generation of the Austrian School of Economics and highlights the fundamentals principles of the contemporary Austrian Economics. 
INDEX

Mots-clés : histoire de la pensée économique, processus de marché

Schlüsselwörter : geschichte des wirtschaftsdenkens, marktprozess

Keywords : market process, history of economic thought

\section{AUTEUR}

CHRISTEL VIVEL

ESDES, Université catholique de Lyon 